\title{
Phase-bistable Kerr cavity solitons and patterns
}

\author{
Germán J. de Valcárcel ${ }^{1}$ and Kestutis Staliunas ${ }^{2}$ \\ ${ }^{1}$ Departament d' Òptica, Universitat de València, Dr. Moliner 50, 46100 Burjassot, Spain \\ ${ }^{2}$ ICREA and Departament de Física i Enginyeria Nuclear, Universitat Politècnica de Catalunya, Colom 11, 08222 Barcelona, Spain
}

(Received 19 December 2012; published 3 April 2013)

\begin{abstract}
We study pattern formation in a passive nonlinear optical cavity on the basis of the classic Lugiato-Lefever model with a periodically modulated injection. When the injection amplitude sign alternates, e.g., following a sinusoidal modulation in time or in space, a phase-bistable response emerges, which is at the root of the spatial pattern formation in the system. An asymptotic description is given in terms of a damped nonlinear Schrödinger equation with parametric amplification, which allows gaining insight into the basic spatiotemporal dynamics of the system. One- and two-dimensional phase-bistable spatial patterns, such as bright and dark-ring cavity solitons and labyrinths, are demonstrated.
\end{abstract}

DOI: 10.1103/PhysRevA.87.043802 PACS number(s): 42.65.Sf, 42.65.Tg, 05.45.-a, 05.65.+b

\section{INTRODUCTION}

Spatially extended systems brought far from equilibrium can exhibit spontaneous breaking of spatial symmetries, leading to the formation of patterns, both extended (e.g., labyrinths, rolls, or hexagons) and localized (e.g., fronts, vortices, or spatial solitons). Such dissipative structures have been predicted and observed in a variety of nonlinear systems, as diverse as biological, chemical, and physical [1-10], and their study occupies a central part in nonlinear science. In particular, spatial solitons in optical cavities - called cavity solitons for obvious reasons-are receiving permanent attention not only because of their interest in basic nonlinear science but also for their potential applicability in optical information storage and processing [11-15]. Many types of nonlinear optical cavity systems have been considered in this context [16-32], among them optical parametric oscillators, photorefractive oscillators, cavities containing liquid-crystal light valves, absorptive optical bistability, and, outstandingly, semiconductor microcavites [33].

In this paper we address pattern formation in a paradigmatic passive nonlinear optical cavity, which has been considered for a long time in the literature: the Kerr cavity. Such a system consists of an optical resonator containing a dispersive thirdorder nonlinear medium (such as silica), which is illuminated by a coherent light beam the so-called injected signal. As the optical Kerr effect present in the medium modifies its refractive index proportionally to the circulating light intensity, the cavity is in fact a passive nonlinear interferometer. Since the seminal paper by Lugiato and Lefever [34] many other studies have followed that have shown the richness of the model nonlinear dynamics, which includes periodic patterns and cavity solitons [35-40]. The latter can be excitable and can be used as bits for logical operations [41]. Recent experiments are demonstrating some of those predictions $[15,42]$. The present work considers a type of injected signal that differs from previous studies in Kerr cavities, and which endows such systems with relevant properties such as phase bistability (unlike the phase monostability inherent in the Lugiato-Lefever model [34]) and the type of dissipative structures associated with it. Note that bistability between two different amplitudes can be induced by a periodic injection [43], while in the present paper we consider the induction of a phase bistability.
The present article is based on the concept of "rocking" [44], which is a modulation technique converting a phaseinvariant self-oscillatory system into a phase-bistable one. Rocking consists of the forcing of the self-oscillatory system at a frequency close to its natural one (so-called 1:1 resonance) but whose amplitude is not a constant but modulated, unlike the classic periodic forcing. In particular, the modulation must be such that the sign of its amplitude alternates (in time or in space). In the special case of a laser (a paradigm of self-oscillatory systems) rocking consists of injecting a resonant signal into the cavity (a laser with an injected signal) whose amplitude is modulated. Rocking was initially proposed in the form of a sinusoidal, time-modulated additive signal to the complex Ginzburg-Landau (CGL) equation [44]—which is the simplest description of a laser and of any self-oscillatory system close to threshold-and later generalized to different forms of modulation, even random [45]. Such a kind of temporal rocking has been demonstrated experimentally in optical [46] and in electronic [45,47] systems. More recently spatial rocking has been proposed as a universal mechanism [48] and applied to special optical systems (semiconductor lasers) [49], where the injection is periodically modulated in space. Finally, rocking has been considered and experimentally demonstrated not only in spatially extended systems but also in small aspect ratio systems $[50,51]$. The practical use of rocking is twofold: (1) Small aspect ratio self-oscillatory systems transform into phase-bistable systems, i.e., become switchable between two equivalent states whose phases differ by $\pi$, and (2) large aspect ratio self-oscillatory systems transform into pattern-forming systems with the spatial patterns typical to phase-bistable systems.

All previous studies about rocking considered selfoscillatory systems above their oscillation threshold (Hopf bifurcation). After the first proposal [44] it was believed that rocking could impose a phase bistability (and its peculiar pattern formation) only on an already existing nontrivial oscillating state. In fact, above threshold, the dynamics of a laser can be roughly described in terms of a potential $[44,52]$ having the form of a sombrero with an unstable maximum at the off state and a degenerate minimum corresponding to the phase-invariant on state. The rocking breaks the continuous phase symmetry of the on state down to a bistable situation, 
but is unable to break such symmetry below the oscillation threshold. However, that mechanical analogy works only when the nonlinearity of the system is saturating, or absorbing (i.e., real valued or close to real valued), which is the typical situation in most pattern-forming systems in nonlinear optics. Here we propose and show in detail that passive nonlinear optical cavities, whose nonlinearity is dispersive (i.e., imaginary valued or close to imaginary valued) can be effectively driven by rocking as well, leading to a phase-bistable response and to the spatial patterns associated with it. Systems such as those, of practical use in optics, are, e.g., vertical-cavity surface emitting lasers (VCSELs) below the threshold, the compact systems that already proved themselves to generate spatial solitons $[53,54]$. For a given set of parameters those localized structures have a fixed phase (imposed by the phase of the used monochromatic injection $[53,54]$ ), whereas the ones we present here are phase bistable, which can be of interest in the arena of optical information processing.

In the following sections of the article we first consider the model for a dispersive passive nonlinear resonator (the Lugiato-Lefever model [34]), and show that rocking converts it, in a limit, into a parametrically driven dissipative nonlinear Schrödinger (NLS) equation (Sec. II). A further analytical study is performed on the NLS model and compared with the numerics performed on the rocked Lugiato-Lefever model throughout the paper. Comparing the analytical results of the simplified (macroscopic) model with the numerical results on the initial (microscopic) model, we thus prove the correctness of the derivations. These analyses comprise both the homogeneous steady states of the system (Sec. III) and the patterns and spatial structures appearing both for spatial and temporal rocking (Sec. IV), which are followed by our conclusions.

\section{THE MODEL}

We consider the classic Lugiato-Lefever model [34] describing pattern formation in a broad-area passive optical cavity, filled by either a Kerr medium or a highly detuned two-level atomic system,

$$
\partial_{t} E=F-(1+i \eta \theta) E+i \nabla^{2} E+i \eta|E|^{2} E,
$$

where $E(\mathbf{r}, t)$ is the intracavity field complex amplitude, $F$ is the amplitude of injected field, $\eta \theta$ is the normalized detuning between the injection frequency and the cavity resonance frequency (the single longitudinal mode case is considered), $\nabla^{2}=\partial^{2} / \partial x^{2}+\partial^{2} / \partial y^{2}$ is the transverse Laplacian, and $\eta$ is +1 for a self-focusing nonlinearity and -1 for a selfdefocusing one. In (1) all quantities are dimensionless.

We study the following cases:

$$
\begin{aligned}
& \text { temporally modulated injection : } F=F_{0} \cos \left(\omega_{0} t\right) \text {, } \\
& \text { spatially modulated injection : } F=i F_{0} \cos \left(k_{0} x\right),
\end{aligned}
$$

where the amplitude $F_{0}$ is assumed real without loss of generality and the imaginary unit factor in (2b) is used for convenience. Such types of injection can be generated by injecting two plane waves into the optical cavity, either on axis but with unequal frequencies [Eq. (2a)] or with equal frequencies but tilted to the opposite angles with respect to the optical axis of the cavity [Eq. (2b)].
In order to gain insight into the effects of this type of forcing we consider first the case of large and fast injection $\left(F_{0}, \omega_{0}, k_{0} \gg 1\right)$. We note that the use of large injections is not against the uniform-field approximation used to derive Eq. (1) because the relevant field in this respect is the circulating field, and this is not large, as we show below. In this case an approximate treatment is justified which is based on a few-mode expansion of the field, as suggested by the form of forcing. For example, in the temporally modulated case (2a) we write

$$
E(\mathbf{r}, t)=A(\mathbf{r}, t)+B_{+}(\mathbf{r}, t) e^{i \omega_{0} t}+B_{-}(\mathbf{r}, t) e^{-i \omega_{0} t},
$$

where all amplitudes are assumed to be slowly varying; in the case of spatial modulation we just substitute $e^{ \pm i \omega_{0} t} \rightarrow e^{ \pm i k_{0} x}$. Inserting (3) into (1), and neglecting higher harmonics, we arrive at the following coupled equations:

$$
\begin{gathered}
\partial_{t} A=-(1+i \eta \theta) A+i \nabla^{2} A+i \eta|A|^{2} A \\
+2 i \eta\left[\left(\left|B_{+}\right|^{2}+\left|B_{-}\right|^{2}\right) A+B_{+} B_{-} A^{*}\right], \\
\partial_{t} B_{ \pm} \pm i \omega_{0} B_{ \pm}= \\
\quad \frac{1}{2} F_{0}-(1+i \eta \theta) B_{ \pm}+i \nabla^{2} B_{ \pm}+i \eta\left[\left|B_{ \pm}\right|^{2}\right. \\
\left.+2\left(|A|^{2}+\left|B_{\mp}\right|^{2}\right)\right] B_{ \pm}+i \eta A^{2} B_{\mp}^{*} .
\end{gathered}
$$

If $\omega_{0}$ and $F_{0}$ are large, then the amplitudes $B_{ \pm}$can be adiabatically eliminated from (4b) as $B_{ \pm}=\mp \frac{i}{2}\left(F_{0} / \omega_{0}\right)$, and the equation for $A$ becomes

$$
\partial_{t} A=-[1+i \eta(\theta-2 \gamma)] A+i \eta \gamma A^{*}+i \nabla^{2} A+i \eta|A|^{2} A \text {, }
$$

which is the searched order parameter equation. In (5) we defined the composite rocking parameter $\gamma=\frac{1}{2}\left(F_{0} / \omega_{0}\right)^{2}$, which is one of the main parameters of the model. Note that the total field is $E(\mathbf{r}, t)=\sqrt{2 \gamma} \sin \left(\omega_{0} t\right)+A(\mathbf{r}, t)$.

In the case of a spatially modulated injection, Eq. (2b), one obtains the same Eq. (4a) for $A$ and equations similar to Eq. (4b) for $B_{ \pm}$. In this case $k_{0}$ and $F_{0}$ are assumed to be large and the amplitudes $B_{ \pm}$can be adiabatically eliminated such as in the case of temporal rocking, now as $B_{ \pm}=\frac{1}{2}\left(F_{0} / k_{0}^{2}\right)$. The equation for $A$ reduces exactly to (5), now with $\gamma=$ $\frac{1}{2}\left(F_{0} / k_{0}^{2}\right)^{2}$. The total field in this case of spatial rocking is $E(\mathbf{r}, t)=\sqrt{2 \gamma} \cos \left(k_{0} x\right)+A(\mathbf{r}, t)$.

We note that we obtain exactly the same results by applying a standard multiple-scale analysis [55] in which $F_{0}, \omega_{0}, k_{0}^{2}=$ $O\left(\varepsilon^{-1}\right)$, where $0<\varepsilon \ll 1$ is a smallness parameter, and the field is expanded as $E(\mathbf{r}, t)=E_{0}(\mathbf{r}, t, T)+\varepsilon E_{1}(\mathbf{r}, t, T)+$ $O\left(\varepsilon^{2}\right)$, where $T=\varepsilon^{-1} t$ is a fast time scale (see Refs. [43,47] for more details).

Hence both types of injection, as represented by (2), lead to the field $E$ composed of two terms: one that follows the form of forcing either in time or in space, and another one $(A)$, which is a dynamical field ruled by macroscopic Eq. (5). We note that in the spatial rocking case we derived (5) for a one-dimensional (1D) forcing. If the modulation is truly 2D (e.g., of the form $\left.F=i F_{0}\left[\cos \left(k_{0} x\right)+\cos \left(k_{0} y\right)\right]\right)$ the dynamical equation then has the same form (5), however, the rocking parameter is to be generalized as $\gamma=\left\langle\left|E_{F}\right|^{2}\right\rangle=\left\langle E_{F}^{2}\right\rangle$, where $E_{F}$ is the solution to $i \nabla^{2} E_{F}=-F$, and \langle\rangle denotes a spatial average (see Ref. [48] for more details).

Equation (5) is a damped NLS equation with a phasesensitive gain (the term $i \eta \gamma A^{*}$ ), which is responsible for 
the predicted phase bistability: As the only phase symmetry supported by Eq. (5) is the discrete one, $A \rightarrow-A$, if $A$ is a solution to (5), then $-A$ is solution as well and both solutions have exactly the same dynamical properties; in particular, if $A$ is stable, then $-A$ is stable as well. Equation (5) finds applications in many different contexts, such as nonlinear optics or the parametric excitation of waves in chains of coupled pendula, fluids, plasmas, and many others (see Refs. [2,8,56-58] and references therein), and has been considered many times in the literature, thanks to which many properties of its solutions are known.

\section{HOMOGENEOUS STEADY STATES AND THEIR STABILITY}

Equation (5) admits two types of homogeneous steady states: (i) the off state $A=A_{0}=0$, and (ii) the on (or rocked) states $A=A_{ \pm}=a_{ \pm} \exp \left(i \phi_{ \pm}\right)$, with $a_{ \pm}^{2}=\theta-2 \gamma \pm$ $\sqrt{\gamma^{2}-1}$ and $\gamma \exp \left(2 i \phi_{ \pm}\right)=\mp \sqrt{\gamma^{2}-1}+i \eta$. Note that the last expression leads to two values for $\phi_{ \pm}$(for each sign \pm ) differing by $\pi$, as predicted: phase-bistable states. It is easy to show that the rocked state $A_{-}$is always unstable, therefore we discard it from our analysis. The rocked state $A_{+}$exists for $\theta>\sqrt{3}$ and in that case it extends from $\gamma=\gamma_{\min }$ to $\gamma=$ $\gamma_{\max }=\left(2 \theta+\sqrt{\theta^{2}-3}\right) / 3$. The expression for $\gamma_{\text {min }}$ depends on the detuning $\theta$ : For $\sqrt{3}<\theta<2, \gamma_{\min }=\left(2 \theta-\sqrt{\theta^{2}-3}\right) / 3$, whereas for $\theta>2, \gamma_{\min }=1$. At $\gamma=\gamma_{\max }, A_{+}=0$, which corresponds to a supercritical pitchfork bifurcation from the off state. The line at which $A_{-}=0$ marks another (subcritical) pitchfork bifurcation that exists whenever $\theta>2$ and occurs at $\gamma=\gamma_{0}=\left(2 \theta-\sqrt{\theta^{2}-3}\right) / 3$. Hence when $\theta>2$, the off state and the rocked states coexist for $1<\gamma<\gamma_{0}$. Figure 1 summarizes the above, where we include the existence domains of these spatially uniform locked states as follows from simulating Eq. (1) in the case of temporal rocking (2a) with $\omega_{0}=2 \pi$ (we set $\nabla^{2} E \rightarrow 0$ as here we consider only the

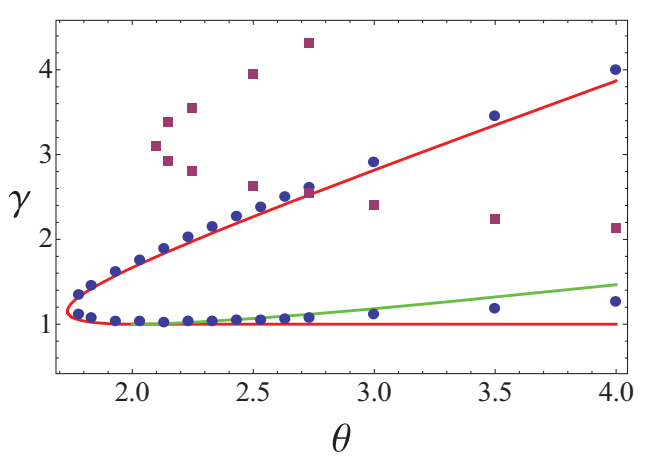

FIG. 1. (Color online) Domains of existence of homogeneous steady states. The curves correspond to analytical predictions from Eq. (5): $\gamma_{\max }$ (upper: red), $\gamma_{\min }$ (lower: red), and $\gamma_{0}$ (middle: green). The off state is stable outside the tongue formed by the upper and middle curves. The rocked state $A_{+}$exists and is stable between the upper and lower curves. Between the middle and the lower curves the off state and the rocked state are coexisting according to (5). Symbols denote boundaries obtained from the numerical integration of Eq. (1) considering the temporal rocking with parameters $\omega_{0}=2 \pi$ and $F_{0}=\omega_{0} \sqrt{2 \gamma}$; see main text. existence of spatially homogeneous states, not their stability; see below).

We numerically find two tongues of stable rocking, the lower one showing a perfect agreement with the theory based on the order parameter equation (5). The upper tongue corresponds to a different branch of rocked states, which are beyond the description by the simple mean field model (5). Here we just note that the position of the upper tongue depends on the rocking frequency $\omega_{0}$, i.e., not only on the composite parameter $\gamma=\frac{1}{2}\left(F_{0} / \omega_{0}\right)^{2}$, and in particular is most promoted for moderate and small values of $\omega_{0}$. We remind that (5) is derived in the limit $\omega_{0}, F_{0} \rightarrow \infty$.

The linear stability analysis of the off state of (5) against spatially dependent perturbations of the form $\exp (\lambda t+i \mathbf{k} \cdot \mathbf{r})$ yields $\lambda=-1 \pm \sqrt{\gamma^{2}-\left(\eta \Delta+k^{2}\right)^{2}}$, where $\Delta=\theta-2 \gamma$ and $k=|\mathbf{k}|$. The maximum of $\lambda$ occurs at $k^{2}=k_{c}^{2}=-\eta \Delta$ if $\eta \Delta<0$ or at $k=0$ if $\eta \Delta>0$. Thus the instability scenario depends on the nonlinear focusing parameter $\eta$. If $\eta=+1$ (self-focusing nonlinearity) the off solution loses stability at any detuning $\theta$ by increasing $\gamma$ : (i) For $\theta<2$ the bifurcation is towards $k=k_{c}=\sqrt{2-\theta}$ and occurs at $\gamma=1$; (ii) for $\theta>2$ the bifurcation is towards $k=0$ and occurs at $\gamma=\gamma_{0}$. On the contrary, if $\eta=-1$ (defocusing nonlinearity), the off solution is unstable only within the outer tongue in Fig. 1, formed by the upper and lower curves, which extends for $\theta>\sqrt{3}$ : (i) At $\gamma=\gamma_{\max }$ the bifurcation is towards $k=0$ for any $\theta$; (ii) for $\sqrt{3}<\theta<2$ the bifurcation is again towards $k=0$ and occurs at $\gamma=\gamma_{\min }$; and (iii) for $\theta>2$ the bifurcation is towards $k=k_{c}=\sqrt{\theta-2}$ and occurs at $\gamma=1$.

The above stability analysis of the NLS equation (5) is summarized in Fig. 2, where the insets show the numerical stability analysis of the off state. For that purpose we integrated (1), by permanently adding weak, white noise, delta correlated in space and in time, using as the initial condition the off state $E=0$. Growing modes in the wave-number domain indicate instability: The modes growing on a ring indicate spatial instability, with the radius corresponding to the wave number of the maximally growing spatial mode, $k \approx k_{c}$. The spot appearing at the center of the wave-number domain evidences the on-axis instability, $k \approx 0$. The numerical results from the full model (1) correspond well to theoretical conclusions from the reduced model (5).

A similar analysis performed in the case of the rocked state $A_{+}$leads to the following expression for the eigenvalues: $\lambda=$ $-1 \pm \sqrt{1-\left(k^{2}+2 \mu\right)\left[k^{2}+2(\mu+\Delta)\right]}$, where $\mu=\sqrt{\gamma^{2}-1}$. The analysis is less involved in this case. In the self-defocusing case $\eta=-1$, the rocked state is stable across its entire existence domain. In the self-focusing case $\eta=+1$ the situation is reversed: The rocked state is unstable across its entire existence range, thus, in this case, only patterns (extended or localized) are expected.

Figure 3 shows the stable rocked states in both cases of temporal and spatial rocking. The parameters in both cases were chosen to result in approximately the same value of the rocking parameter, $\gamma=\frac{1}{2}\left(F_{0} / \omega_{0}\right)^{2}=\frac{1}{2}\left(F_{0} / k_{0}^{2}\right)^{2}$. The expected result is obtained: the coexistence of relatively homogeneous domains of opposite phases. In the case of temporal rocking the field was averaged in time over one spatial period, which eliminates the fast varying part of the field, $E(\mathbf{r}, t)=\sqrt{2 \gamma} \sin \left(\omega_{0} t\right)+A(\mathbf{r}, t)$, and allows representing 

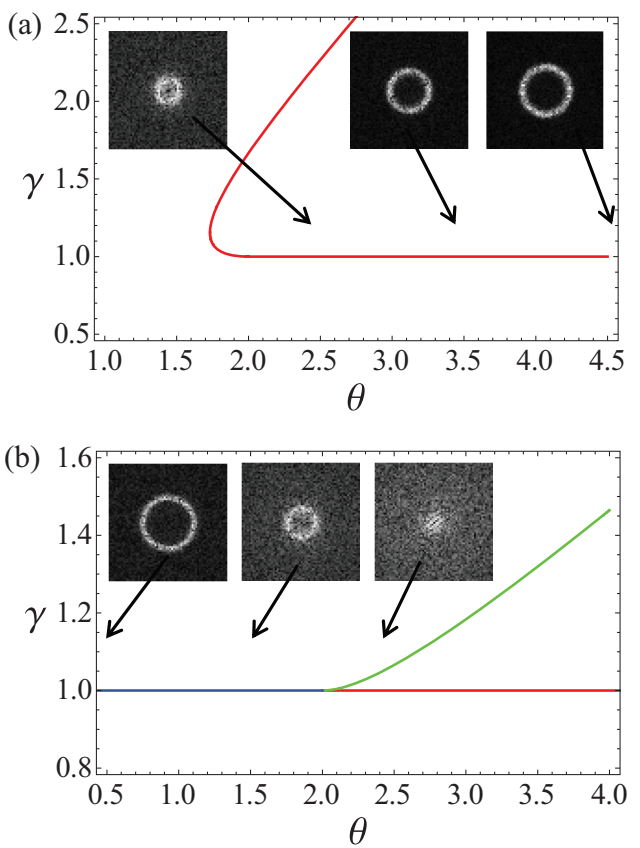

FIG. 2. (Color online) Stability of the off state. (a) Defocusing nonlinearity: The off state is stable outside the tongue; increasing $\gamma$ the bifurcation is towards $k=k_{c}=\sqrt{\theta-2}$ for $\theta>2$; along the rest of the stability boundary the bifurcation is towards $k=0$. (b) Self-focusing nonlinearity: For $\theta<2$ (blue curve) the bifurcation is towards $k=k_{c}=\sqrt{2-\theta}$ and occurs at $\gamma=1$; for $\theta>2$ the bifurcation is towards $k=0$ and occurs at $\gamma=\gamma_{0}$ (green curve); above the blue and green curves patterns exist, and between the green and the red curves bright solitons exist. The insets indicate the initial stages of the instability of the off state in the far field, as obtained by integrating (1) (numerical stability analysis), with parameters $F_{0}=9.5$ and $\omega_{0}=2 \pi(\gamma \approx 1.14)$ on an integrating window of size 70 ; the far field is displayed on a window of size $6(-3<k<+3)$. Integration time is 50 .

only the slow component $A(\mathbf{r}, t)$. In case of spatial rocking an analogous averaging in space has been done. Technically we performed the averaging by filtering out all the field components in the far field with $|\mathbf{k}|>k_{0} / 2$. For comparison, also the total (unfiltered) field $E(\mathbf{r}, t)=\sqrt{2 \gamma} \cos \left(k_{0} x\right)+A(\mathbf{r}, t)$ is presented in Fig. 3(b) in the case of spatial rocking.

\section{SPATIAL STRUCTURES}

It is known that Eq. (5) admits several types of localized structures (cavity solitons in our case) that have been the subject of abundant literature. The nature of the localized structures depends on the nonlinear focusing parameter $\eta$.

In the self-defocusing case $\eta=-1,1 \mathrm{D}$ localized structures are kinks joining the two stable rocked states of opposite phase; they appear as dark lines separating two illuminated domains and hence are called dark cavity solitons, for their resemblance to the ones in the NLS equation. In 2D they can form so-called phase domains, as shown above in Fig. 3. The straight domain boundaries have the analytic form $A=$ $\pm a_{+} \tanh \left(a_{+} x / \sqrt{2}\right) \exp \left(i \phi_{+}\right)$[59], where $a_{+}$and $\phi_{+}$have the same meaning as in the rocked state $A_{+}$. These 1D cavity solitons are stable everywhere the rocked states exist [inside (a)
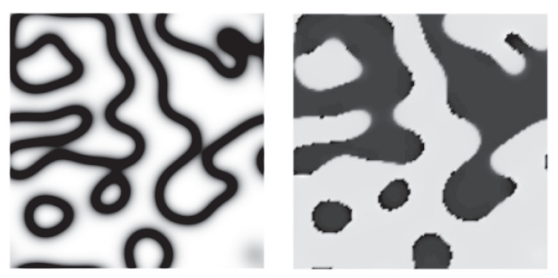

(b)
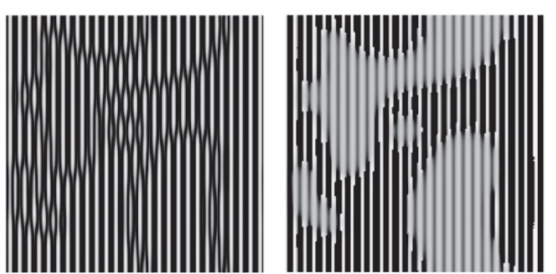

(c)
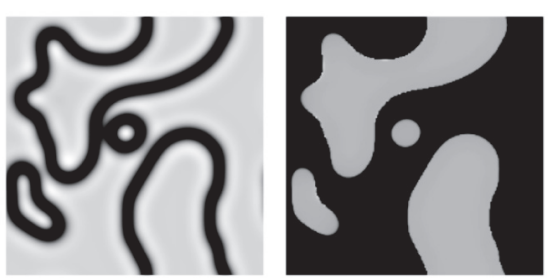

FIG. 3. Field domains of opposite phases obtained from Eq. (1) for $\eta=-1$ (defocusing nonlinearity) in the cases of temporal (a) and spatial (b), (c) rocking. The left column represents the field amplitudes and the right column shows phases. In the case of temporal rocking (a) the field has been averaged over one period of injection. In the case of spatial rocking the full field (b) is shown, as well as the field averaged in space (c) (see main text for a description of averaging). Parameters: space window size $l=70$, detuning $\theta=2.5$, rocking parameter $\gamma=1.13$. Microscopic rocking parameters are $F_{0}=9.5$, $\omega_{0}=2 \pi$ for temporal rocking, and $F_{0}=9.5, k_{0}^{2}=2 \pi$ for spatial rocking.

the tongue in Fig. 2(a)] [60]. In 2D an interesting issue is the evolution of curved dark lines [61-63]. Typically the curved segments expand, or contract, depending on the parameters $\gamma, \theta$. (We note that since both rocked states are equivalent, then the straight domain boundaries do not move: They are always at the Maxwellian point.) The contracting dark lines either end up as straight lines (for semi-infinite phase domains) or disappear (for closed domains). The expanding domains grow, and form a labyrinthlike pattern. Figures 4(a) and 4(b) show labyrinths for both temporal and spatial rocking. In a narrow parameter range between the expansion and contraction of domains the so-called dark-ring solitons can appear in 2D case, which consist of a small circular region, whose phase is not uniform but is close to one of the steady states, $\phi_{+}$, surrounded by the homogeneous state of the opposite phase. The phase jump from the outer region to the inner one is accompanied by a zero of the field and then these solitons appear as dark rings [64]. For the parametrically driven Ginzburg-Landau equation [62] and for the Swift-Hohenberg equation [61] the existence region of dark-ring solitons is very narrow, as the opposite fragments of the dark ring interact very weakly. For more realistic systems such as optical parametric oscillators [65] the dark-ring solitons have relatively large stability areas, as the domain boundaries possess strongly oscillatory tails, which mutually lock the opposite segments of the dark ring. 
(a)

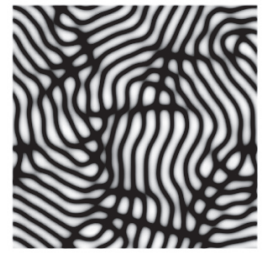

(c)

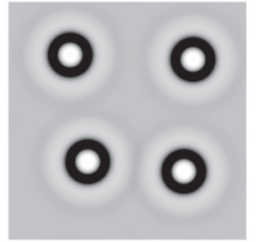

(b)

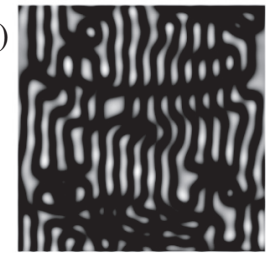

(d)

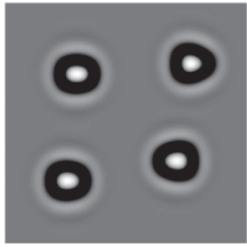

FIG. 4. Labyrinth patterns (a), (b), and dark-ring solitons (c), (d) for spatial and temporal rocking, as obtained by numerical integration of (1) in the self-defocusing case $\eta=-1$. The parameters are as in Fig. 3, except for detuning: For labyrinths (a), (b) the detuning is $\theta=5.0$, and for dark-ring solitons (c), (d) the detuning was chosen as $\theta=2.8$. The averaged (in time and space for temporal and spatial rocking, respectively) fields are plotted.

Examples of stable dark-ring solitons are shown in Figs. 4(c) and 4(d) both for temporal and spatial rocking.

We note that these cavity solitons (dark, in 1D, or dark ring, in 2D) are specific of phase-bistable systems $[10,56,64,66]$ as it is the dynamical equivalence between the two oppositely phased states that allows for the stability of such structures. We note, however, that these phase-bistable structures can be affected by instabilities, such as the nonequilibrium IsingBloch transition [56,66-68], leading to their motion across the transverse plane.

In the self-focusing case, $\eta=+1$, different localized structures can be found, which are bell shaped, and resemble bright cavity solitons. In $1 \mathrm{D}$ they have the form $A= \pm \sqrt{2} a_{+} \operatorname{sech}\left(a_{+} x\right) \exp \left(i \phi_{+}\right)$[59] (note the presence of the two signs, \pm , accounting for phase bistability), where $a_{+}$and $\phi_{+}$have again the same meaning as in the rocked state $A_{+}$. Unlike the previous case these 1D cavity solitons are stable within a region inside the "triangle" in Fig. 2(a), close to $\theta=2$ [69-72]. In 2D the bright cavity solitons have the form $A(\mathbf{r})=a_{+} R\left(a_{+} r\right) \exp \left(i \phi_{+}\right)$, where the radial function is the bell-shaped nodeless solution to the ordinary differential equation $R^{\prime \prime}(r)+r^{-1} R^{\prime}(r)-R(r)+R(r)^{3}=0$ [73]. This soliton exists in the same region as its $1 \mathrm{D}$ analog and is affected as well by a Hopf bifurcation, whose location differs from the $1 \mathrm{D}$ case, which limits its stability. The bifurcation can be determined again by means of numerical methods only [33]. The conclusion is that truly $2 \mathrm{D}$, phase-bistable bright solitons (bright spots of light surrounded by darkness) can be excited in a parametric region on the plane of control parameters $(\theta, \gamma)$ close above $\gamma=1$ and in a narrow range around $\theta=2$.

Numerically, by integrating the full model, we were able to excite the bright solitons in the case of temporal rocking, as shown in Fig. 5. In the spatial rocking case the bright solitons, however, appeared unstable. The main reason is that the limited size of the grid does not allow to separate completely the slow and fast space scales, those responsible for the spatial envelopes of soliton and the sinusoidal shape
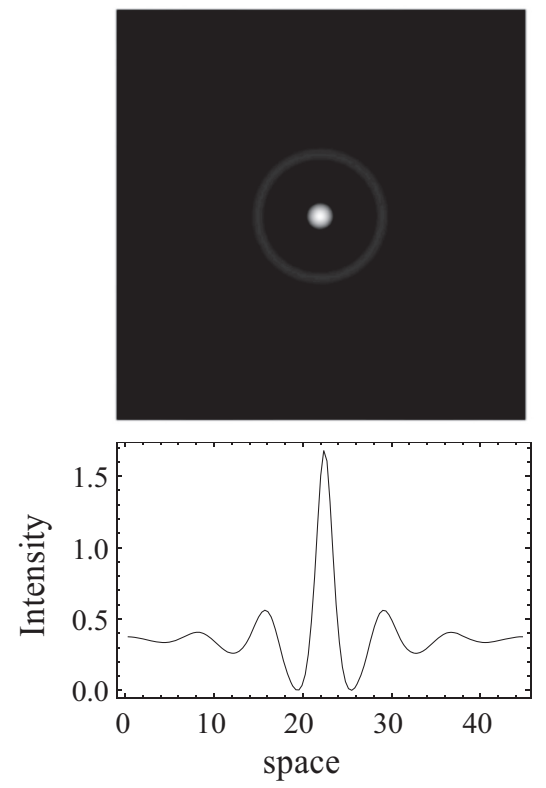

FIG. 5. Bright spatial soliton by temporal rocking, as obtained by numerical integration of (1) in the self-focusing case $\eta=+1$, together with its cross section. The parameters are $\theta=2.1, F_{0}=12$, $\omega_{0}=8(\gamma=1.13)$. Note that the spatial window has been chosen to be of different size from those in Figs. 3 and 4.

of injection for spatial rocking, respectively. The small spatial scale modulation perturbs the soliton, and, since the stability range of soliton is extremely small, destabilizes it.

\section{CONCLUSIONS}

We have shown that temporal and spatial rocking (i.e., the injection of a sinusoidally modulated, in time or space, signal) can drastically change the properties of the Kerr cavity model. In particular, a phase-bistable response emerges, which is at the root of the spatial pattern formation in the system.

An asymptotic description of the problem has been given in terms of a damped nonlinear Schrödinger equation with parametric amplification, Eq. (5). Remarkably, such an equation captures the basic properties of the original system in some order of approximation, including the phase bistability and the emergence of phase domains, labyrinths and (phasebistable) bright and dark solitons. We note that the classic Lugiato-Lefever model with self-focusing nonlinearity already supports 2D spatial patterns [34] and cavity solitons [37] whose phase is uniquely locked to that of the injection. In our case, however, patterns and solitons are phase bistable, which is a fundamental difference from the perspective of information storage and processing as they allow ternary logic (off, plus, and minus states) in contrast to usual binary logic (off and on) in bistable systems.

We have as well shown that some specific dynamics is beyond that simplified model, especially when we depart from the assumptions of strong and fast rocking which led to (5) and hence to describe the net effect of rocking by a single compound parameter $\gamma=\frac{1}{2}\left(F_{0} / \omega_{0}\right)^{2}$ (temporal rocking) or $\gamma=\frac{1}{2}\left(F_{0} / k_{0}^{2}\right)^{2}$ (spatial rocking). For moderate rocking, i.e., $F_{0}, \omega_{0}, k_{0}^{2}=O(1)$, the adiabatic elimination of 
sideband components is not legitimate and their dynamics must play a role, which should be at the roots of the presence of several branches of solutions, as the second rocking tongue in Fig. 1. The analysis of all these interesting phenomena is, however, beyond the scope of this article, which is to be a proof of principle of the excitation of phase bistability and phase-bistable patterns in a Kerr cavity model.

We hope that these results can have an application in two types of already existing experiments. On the one hand, 1D cavity solitons have been recently demonstrated in a Kerrlike optical cavity [42] with a noninstantaneous nonlinearity, and we argue that spatial rocking should be effective as well as temporal rocking, whenever the time scale of rocking is fast as compared with the time scale of the nonlinearity. On the other hand, injected vertical-cavity surface emitting lasers below threshold $[33,53,54]$ are most promising devices for the generation and use of optical cavity solitons and display an effective nonlinearity with a large imaginary (dispersive) part.

\section{ACKNOWLEDGMENTS}

We gratefully acknowledge fruitful discussions with E. Roldán. The Spanish Government and European Union FEDER supported this work through projects FIS2011-26960 and FIS2011-29731-C02-01.
[1] G. Nicolis and I. Prigogine, Self-Organization in Nonequilibrium Systems: From Dissipative Structures to Order through Fluctuations (Wiley, New York, 1977).

[2] M. C. Cross and P. C. Hohenberg, Rev. Mod. Phys. 65, 851 (1993).

[3] M. Cross and H. Greenside, Pattern Formation and Dynamics in Nonequilibrium Systems (Cambridge University Press, New York, 2009).

[4] J. D. Murray, Mathematical Biology (Springer, Berlin, 2002), Vols. I and II

[5] H. Meinhardt, Models of Biological Pattern Formation (Academic, London, 1982).

[6] P. Manneville, Dissipative Structures and Weak Turbulence (Springer, Berlin, 1995).

[7] L. M. Pismen, Patterns and Interfaces in Dissipative Dynamics (Springer, Berlin, 2006).

[8] D. Walgraef, Spatio-Temporal Pattern Formation (Springer, New York, 1997).

[9] P. Mandel, Theoretical Problems in Cavity Nonlinear Optics (Cambridge University Press, New York, 2005).

[10] K. Staliunas and V. J. Sánchez-Morcillo, Transverse Patterns in Nonlinear Optical Resonators (Springer, Berlin, 2003).

[11] A. Esteban-Martín et al., Opt. Express 13, 3631 (2005).

[12] U. Bortolozzo and S. Residori, Phys. Rev. Lett. 96, 037801 (2006).

[13] M. Hisaka and K. Yoshida, Appl. Phys. Lett. 93, 241103 (2008).

[14] F. Pedaci et al., Appl. Phys. Lett. 92, 011101 (2008).

[15] F. Leo et al., Nat. Photonics 4, 471 (2010).

[16] W. J. Firth and C. O. Weiss, Opt. Photonics News 13, 54 (2002).

[17] L. A. Lugiato, IEEE J. Quantum Electron. 39, 193 (2003).

[18] T. Ackemann and W. J. Firth, in Dissipative Solitons, Lecture Notes in Physics Vol. 661 (Springer, Berlin, 2005), p. 55.

[19] Dissipative Solitons: From Optics to Biology and Medicine, edited by N. N. Akhmediev and A. Ankiewicz, Lecture Notes in Physics Vol. 751 (Springer, Berlin, 2008).

[20] D. W. McLaughlin, J. V. Moloney, and A. C. Newell, Phys. Rev. Lett. 51, 75 (1983).

[21] N. N. Rosanov and G. V. Khodova, J. Opt. Soc. Am. B 7, 1057 (1990).

[22] G. S. McDonald and W. J. Firth, J. Opt. Soc. Am. B 7, 1328 (1990).

[23] M. Tlidi, P. Mandel, and R. Lefever, Phys. Rev. Lett. 73, 640 (1994).
[24] M. Brambilla, L. A. Lugiato, and M. Stefani, Europhys. Lett. 34, 109 (1996).

[25] W. J. Firth and A. J. Scroggie, Phys. Rev. Lett. 76, 1623 (1996).

[26] V. J. Sánchez-Morcillo, E. Roldán, G. J. de Valcárcel, and K. Staliunas, Phys. Rev. A 56, 3237 (1997); G. J. de Valcárcel, E. Roldán, and K. Staliunas, Opt. Commun. 181, 207 (2000).

[27] S. Longhi, Opt. Commun. 149, 335 (1998).

[28] K. Staliunas, Phys. Rev. Lett. 81, 81 (1998).

[29] M. Tlidi and P. Mandel, Phys. Rev. Lett. 83, 4995 (1999).

[30] M. Brambilla, T. Maggipinto, G. Patera, and L. Columbo, Phys. Rev. Lett. 93, 203901 (2004).

[31] Y. Tanguy, T. Ackemann, W. J. Firth, and R. Jager, Phys. Rev. Lett. 100, 013907 (2008).

[32] S. D. Jenkins, F. Prati, L. A. Lugiato, L. Columbo, and M. Brambilla, Phys. Rev. A 80, 033832 (2009).

[33] S. Barland et al., Nature (London) 419, 699 (2002).

[34] L. A. Lugiato and R. Lefever, Phys. Rev. Lett. 58, 2209 (1987).

[35] A. J. Scroggie et al., Chaos Solitons Fractals 4, 1323 (1994).

[36] W. J. Firth and A. Lord, J. Mod. Opt. 43, 1071 (1996).

[37] W. J. Firth et al., J. Opt. Soc. Am. B 19, 747 (2002).

[38] D. Gomila, A. Scroggie, and W. Firth, Physica D 227, 70 (2007).

[39] G. Kozyreff, Physica D 241, 939 (2010).

[40] G. Kozyreff and L. Gelens, Phys. Rev. A 84, 023819 (2011).

[41] A. Jacobo et al., New J. Phys. 14, 013040 (2012).

[42] V. Odent, M. Taki, and E. Louvergneaux, New J. Phys. 13, 113026 (2011)

[43] A. V. Gainer and G. I. Surdutovich, Phys. Status Solidi B 150, 539 (1988).

[44] G. J. de Valcárcel and K. Staliunas, Phys. Rev. E 67, 026604 (2003).

[45] K. Staliunas, G. J. de Valcárcel, J. M. Buldú, and J. GarcíaOjalvo, Phys. Rev. Lett. 102, 010601 (2009).

[46] A. Esteban-Martín, M. Martinez-Quesada, V. B. Taranenko, E. Roldán, and G. J. de Valcárcel, Phys. Rev. Lett. 97, 093903 (2006).

[47] J. M. Buldú et al., Chaos 16, 043126 (2006).

[48] G. J. de Valcárcel and K. Staliunas, Phys. Rev. Lett. 105, 054101 (2010).

[49] M. Radziunas and K. Staliunas, Europhys. Lett. 95, 14002 (2011).

[50] K. Staliunas, G. J. de Valcárcel, and E. Roldán, Phys Rev. A 80, 025801 (2009). 
[51] S. Kolpakov, F. Silva, G. J. de Valcárcel, E. Roldán, and K. Staliunas, Phys. Rev. A 85, 025805 (2012).

[52] K. Staliunas et al., Opt. Commun. 268, 160 (2006).

[53] D. Michaelis, U. Peschel, and F. Lederer, Phys. Rev. A 56, R3366 (1997).

[54] L. Spinelli, G. Tissoni, M. Brambilla, F. Prati, and L. A. Lugiato, Phys. Rev. A 58, 2542 (1998).

[55] A. H. Nayfeh, Perturbation Methods (Wiley, New York, 1973).

[56] P. Coullet, J. Lega, B. Houchmanzadeh, and J. Lajzerowicz, Phys. Rev. Lett. 65, 1352 (1990).

[57] P. Coullet and K. Emilsson, Physica D (Amsterdam) 61, 119 (1992).

[58] G. J. de Valcárcel, I. Pérez-Arjona, and E. Roldán, Phys. Rev. Lett. 89, 164101 (2002).

[59] J. W. Miles, J. Fluid Mech. 148, 451 (1984).

[60] I. V. Barashenkov, S. R. Woodford, and E. V. Zemlyanaya, Phys. Rev. Lett. 90, 054103 (2003).

[61] K. Staliunas and V. J. Sánchez-Morcillo, Phys. Lett. A 241, 28 (1998).

[62] D. Gomila, P. Colet, G. L. Oppo, and M. San Miguel, Phys. Rev. Lett. 87, 194101 (2001).
[63] M. Tlidi et al., Opt. Lett. 25, 487 (2000).

[64] V. B. Taranenko, K. Staliunas, and C. O. Weiss, Phys. Rev. Lett. 81, 2236 (1998).

[65] K. Staliunas and V. J. Sánchez-Morcillo, Phys. Rev. A 57, 1454 (1998).

[66] A. Esteban-Martín, V. B. Taranenko, J. García, G. J. de Valcárcel, and E. Roldán, Phys. Rev. Lett. 94, 223903 (2005); Appl. Phys. B 85, 117 (2006).

[67] D. Michaelis, U. Peschel, F. Lederer, D. V. Skryabin, and W. J. Firth, Phys. Rev. E 63, 066602 (2001).

[68] D. V. Skryabin, A. Yulin, D. Michaelis, W. J. Firth, G. L. Oppo, U. Peschel, and F. Lederer, Phys. Rev. E 64, 056618 (2001).

[69] I. V. Barashenkov, M. M. Bogdan, and V. I. Korobov, Europhys. Lett. 15, 113 (1991).

[70] M. Bondila, I. V. Barashenkov, and M. M. Bogdan, Physica D (Amsterdam) 87, 314 (1995).

[71] S. Longhi, G. Steinmeyer, and W. S. Wong, J. Opt. Soc. Am. B 14, 2167 (1997).

[72] V. J. Sánchez-Morcillo et al., Opt. Lett. 25, 957 (2000).

[73] I. V. Barashenkov, N. V. Alexeeva, and E. V. Zemlyanaya, Phys. Rev. Lett. 89, 104101 (2002). 